\title{
A Case of Meningeal Carcinomatosis Due to Signet-Ring Cell Carcinoma That Developed Severe Visual Impairment with Papillary Swelling
}

This article was published in the following Dove Press journal:

International Medical Case Reports Journal

\author{
Shimpei Sugaoka' \\ Takayuki Kanda' \\ Masataka Ito ${ }^{2}$ \\ Masaru Takeuchi $\mathbb{D}^{\prime}$ \\ 'Department of Ophthalmology, National \\ Defense Medical College, Tokorozawa City, \\ Saitama 359-85I3, Japan; ${ }^{2}$ Department of \\ Developmental Anatomy, National Defense \\ Medical College, Tokorozawa City, Saitama \\ 359-85 I3, Japan
}

Purpose: To report a case of meningeal carcinomatosis by metastasis of signet-ring cell carcinoma resulting in severe visual impairment.

Case Presentation: A 49-year-old man visited our hospital complaining of blurred vision in his left eye since 2 months ago. There was no particular history. Best corrected visual acuity (BCVA) was 25/20 in the right eye and 20/20 in the left eye. Papillary swelling in both eyes and disc hemorrhage in the left eye were observed. Gradually, the frequency of dizziness increased, and magnetic resonance imaging (MRI) suggested ventricular enlargement, infiltration around the optic nerve, and meningeal carcinomatosis. Increased cerebrospinal fluid pressure was found, and metastatic adenocarcinoma cells were observed by cerebrospinal fluid cytology. A gastroduodenal biopsy confirmed the diagnosis of poorly differentiated adenocarcinoma with signet-ring cells. Visual acuity at the final visit was 4/200 in both eyes.

Conclusion: If papillary swelling is observed in both eyes, meningeal carcinomatosis should be considered as a differential diagnosis.

Keywords: meningeal carcinomatosis, signet-ring cell carcinoma, papillary swelling

\section{Background}

Meningeal carcinomatosis is a disease with a very poor prognosis in which malignant tumor cells extensively infiltrate into the medullary cavity or meninges via cerebrospinal fluid. Since it is rare that tumor cells are seeded into the optic nerve via cerebrospinal fluid, resulting in optic neuropathy, the characteristics are unclear. We report a case in which meningeal carcinomatosis caused by signet-ring cell carcinoma invaded the optic nerve, resulting in severe visual loss.

\section{Case Presentation}

A 49-year-old man visited an ophthalmology clinic complaining of blurred vision in his left eye since 2 month ago. Optic disc swelling was observed in both eyes, and he was referred to our hospital. At the initial presentation, best corrected visual acuity (BCVA) was 25/20 in the right eye and 20/20 in the left eye. Central flicker was $37 \mathrm{~Hz}$ in the right eye and $36 \mathrm{~Hz}$ in the left eye. Intraocular pressure was $15 \mathrm{mmHg}$ in both eyes. Both eyes had normal light reflexes and negative relative afferent pupillary defect. There was no abnormality in the anterior segment; however, papillary swelling in both eyes and optic disc hemorrhage in the left eye were
Correspondence: Masaru Takeuchi Department of Ophthalmology, National Defense Medical College, 3-2 Namiki, Tokorozawa City, Saitama 359-85I3, Japan Tel +8I-4-2992-I5II

Fax +8I-4-2995-5332

Email masatake@ndmc.ac.jp 


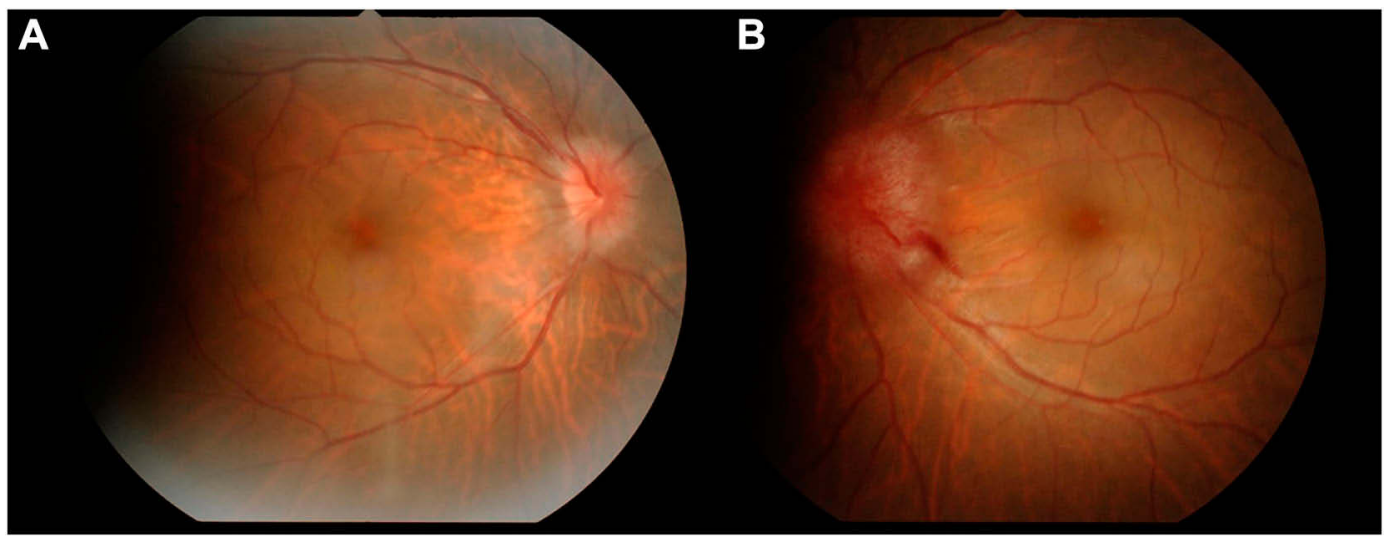

Figure I Color fundus photographs at the initial presentation. Papillary swelling in the right eye (A) and the left eye (B) and optic disc hemorrhage in the left eye are observed.

observed on the fundus examination (Figure 1). nodules on the left frontal skull (Figure 3C), and subcutaFluorescein angiography (FA) revealed dye leakage from the papillae of both eyes (Figure 2A and B) and multiple nodular staining along the veins (Figure 2C). Laboratory examination and chest X-ray were normal, and the tuberculin reaction was weakly positive. Brain CT showed no cerebral ventricular enlargement (Figure 3A), but dilation of the bilateral nerve (Figure 3B), $8 \mathrm{~mm}$ and $10 \mathrm{~mm}$ neous nodules in the right parietal region (Figure 3D) were observed.

Although systemic characteristics of sarcoidosis were not detected, ocular sarcoidosis was the most considered finding from the FA findings. Oral corticosteroids (prednisolone $40 \mathrm{mg} /$ day) were initiated and tapered, but there was no improvement in papillary swelling. The

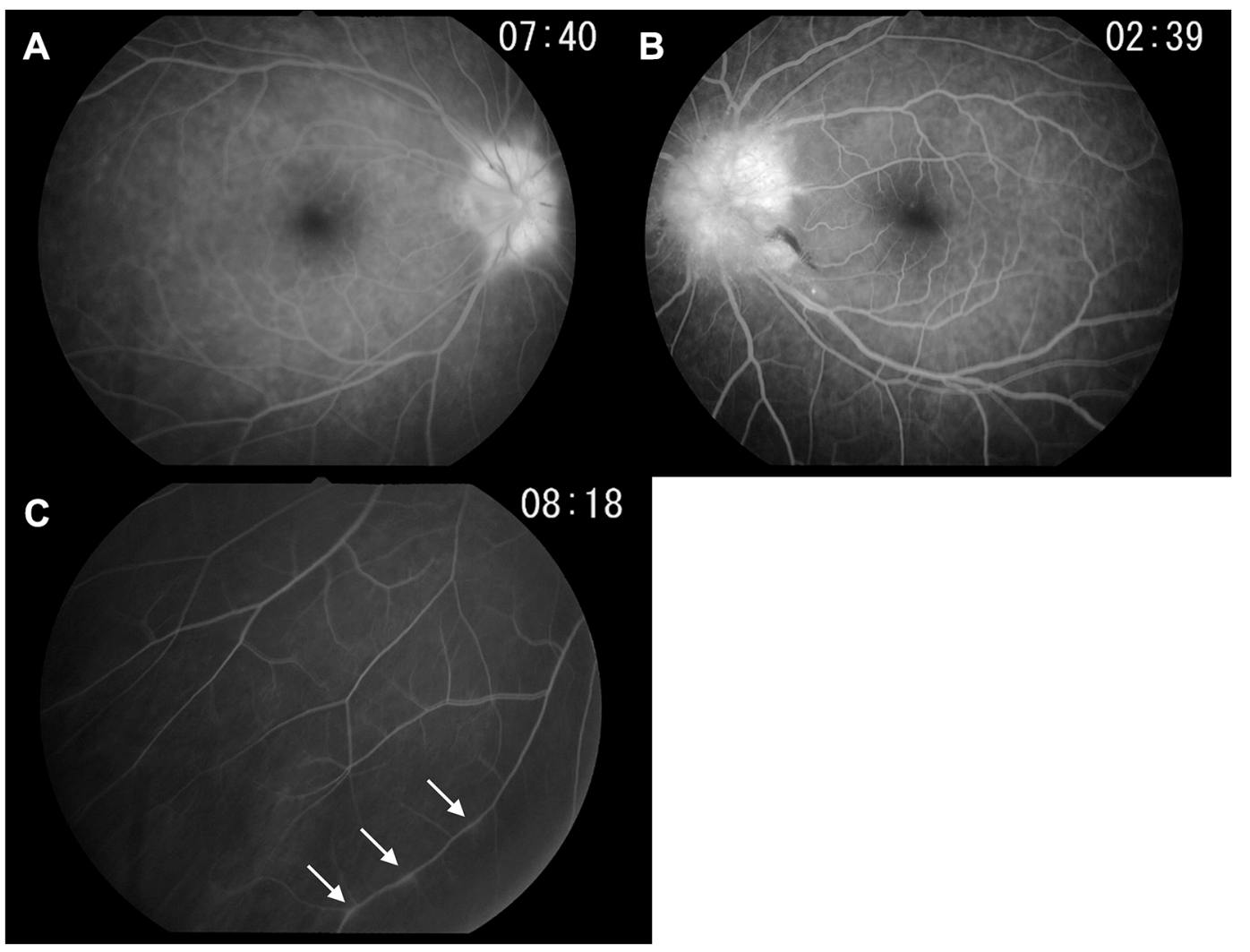

Figure 2 Fluorescein angiography at the initial presentation. Fluorescein angiography revealed dye leakage from the papillae of the right eye (A) and the left eye (B). (C) Multiple nodular staining along the retinal veins was also observed (indicated by white arrows). 


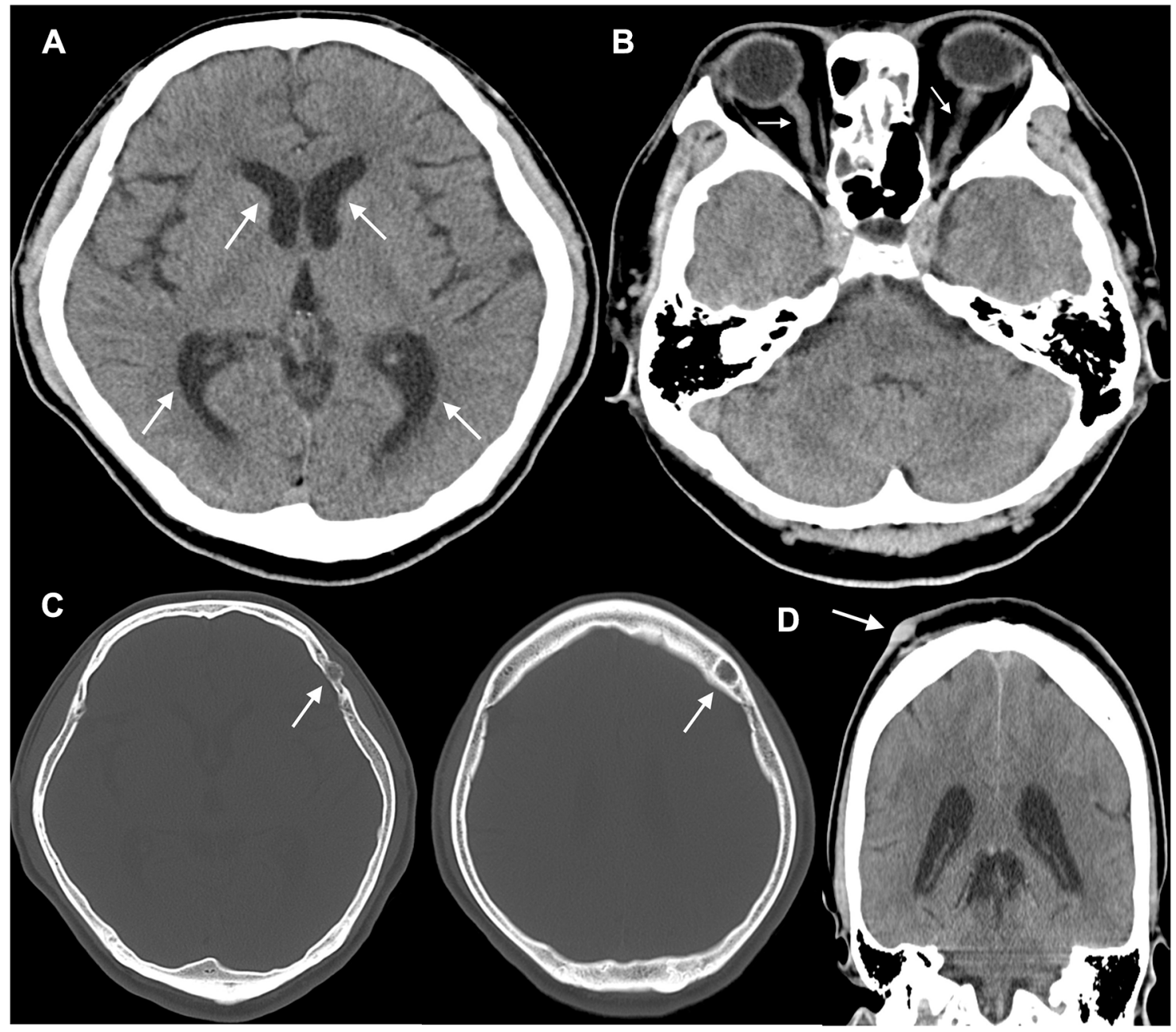

Figure 3 Brain CT findings. (A) No cerebral ventricular enlargement (white arrows), but (B) dilation of the bilateral nerve (white arrows), (C) 8 mm and I0 mm nodules on the left frontal skull (white arrows), and (D) subcutaneous nodules in the right parietal region (white arrow) are detected.

frequency of dizziness further increased 2 months after the first visit. He visited neurosurgery 3 months after the first visit, and it was indicated that he had an abduction disorder in the left eye, right-sided central facial paralysis, mild weakness of the left little finger abductor, and increased tendon reflex in both legs.

In brain/myelography MRI, enhancement of contrast effect around both optic nerves, high signal of fatty tissue around the optic nerve, high signal of cerebral sulcus of the frontal parietal lobe, enlargement of the lateral ventricular angle, and high signal of cervical meninges were recognized (Figure 4). Cerebrospinal fluid examination revealed markedly increased cerebrospinal fluid pressure ( $>30 \mathrm{cmH}_{2} \mathrm{O}$ [not measurable]), cell counts $5 / \mu \mathrm{L}$, cerebrospinal fluid protein $438 \mathrm{mg} / \mathrm{dL}$, and cerebrospinal fluid sugar $45 \mathrm{mg} / \mathrm{dL}$. Cytodiagnosis suggested highly atypical metastatic adenocarcinoma cells (Class IIIb) containing mucus-like substances in the cytoplasm (Figure 5), and biopsy found that poorly differentiated adenocarcinoma with signet-ring cells originating in the gastroduodenum had metastasized to the brain, resulting in meningeal carcinoma, which caused bilateral optic disc invasion. Thereafter, a ventricular abdominal shunt (VP shunt) was performed, and the abduction disorder in the left eye and facial nerve palsy tended to improve. Whole brain irradiation started at another hospital 4 months after the first visit, and chemotherapy (TS-1+cisplatin: SP therapy) started 5 months after the first visit. BCVA at the final visit to our hospital (after 10 months after the first visit) after completing five courses of chemotherapy was 4/200 in both eyes, and central flicker could not be measured in either eye, although bilateral optic disc swelling had improved (Figure 6). Later, his general condition gradually worsened, and he died 16 months after the first visit. 

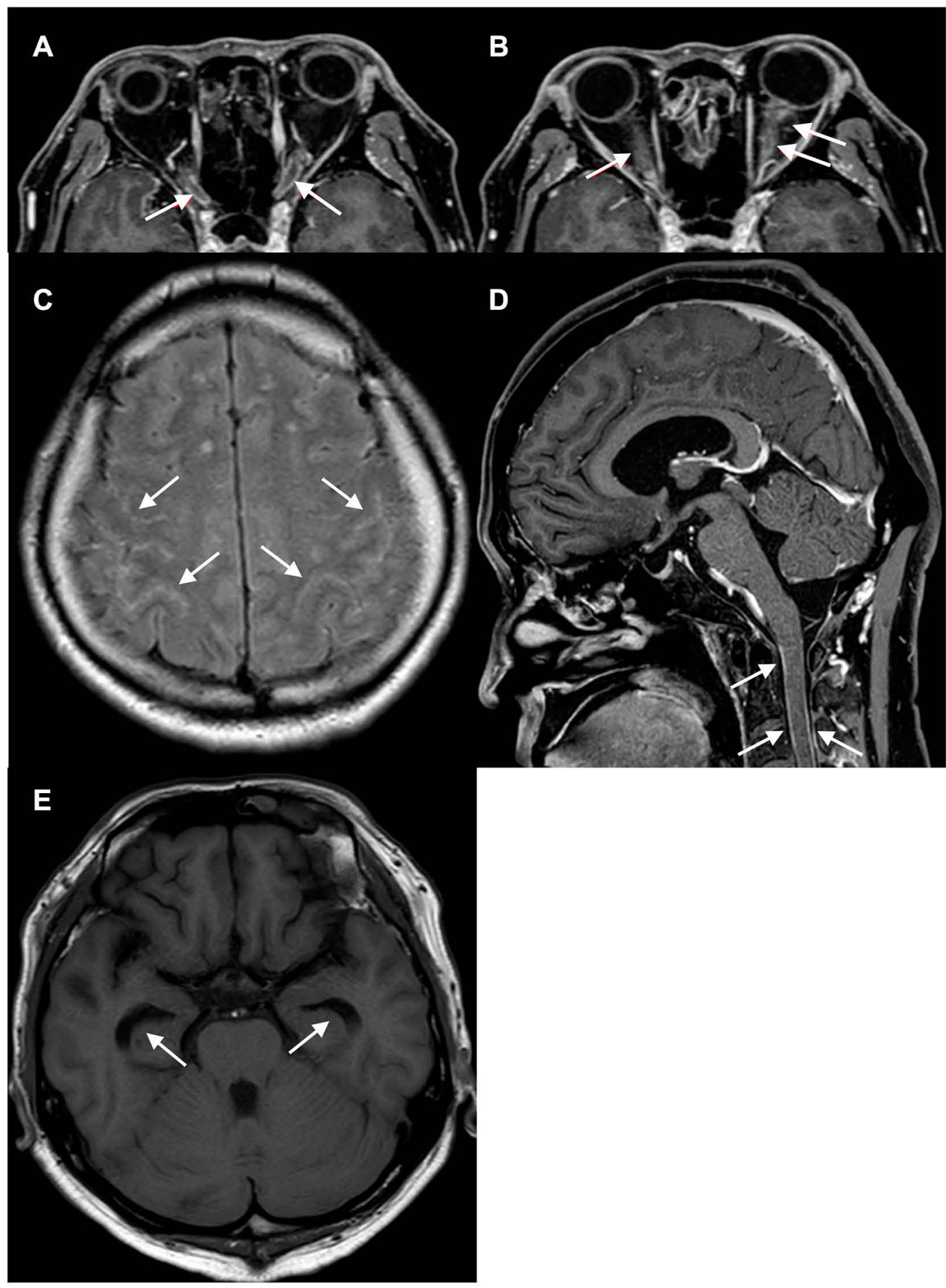

Figure 4 Brain/myelography MRI findings. (A) Enhancement of contrast effect around both optic nerves (white arrows), (B) high signal of fatty tissue around the optic nerve (white arrows), (C) high signal of cerebral sulcus of frontal parietal lobe (white arrows), (D) enlargement of lateral ventricular angle (white arrows), and (E) high signal of cervical meninges (white arrows) are recognized.

\section{Discussion}

Meningeal carcinomatosis develops by cancer cells metastasizing to the brain and spinal cord buffy coat in multiple or diffuse ways. It develops in $5-8 \%$ of metastatic cancers. ${ }^{1-3}$ The most common primary origins of meningeal carcinomatosis in solid tumors are breast cancer, lung cancer, and malignant melanoma in Caucasians. ${ }^{2,4}$ However, the merger frequency of meningeal carcinomatosis in signet-ring cell carcinoma is low, at $0.16-0.69 \%{ }^{5}$ The clinical symptoms of meningeal carcinomatosis are diverse. Most of 


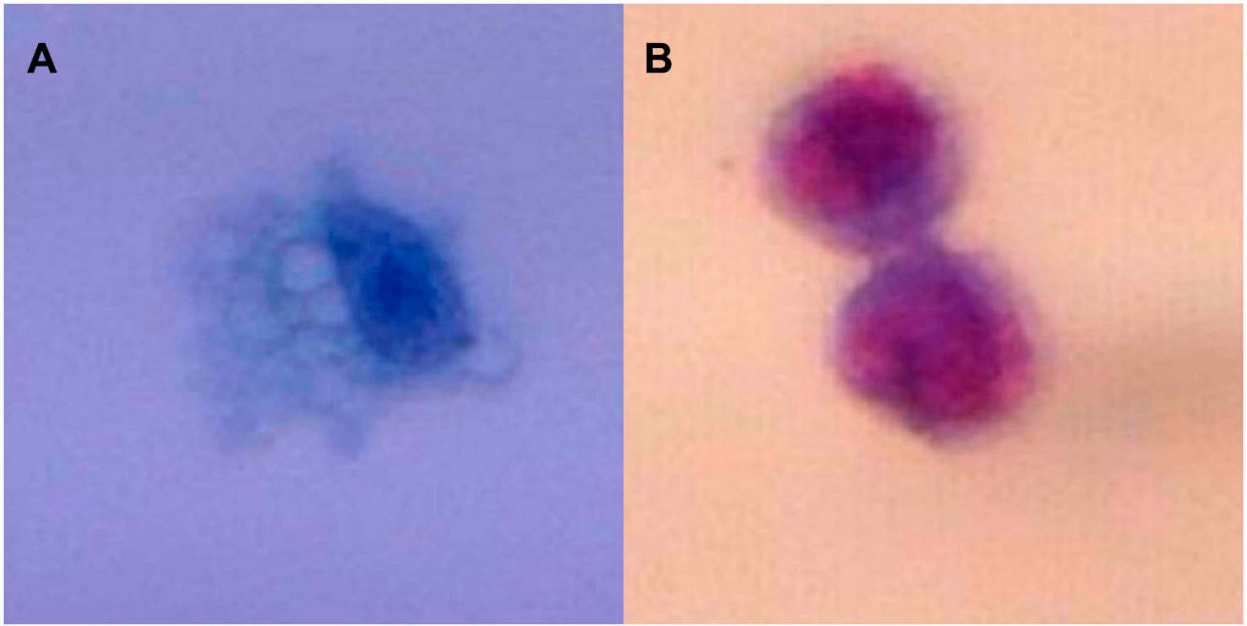

Figure 5 Histopathological examination. (A) Papanicolaou staining and (B) Giemsa staining reveal highly atypical metastatic adenocarcinoma cells (Class Illb) containing mucus-like substances.

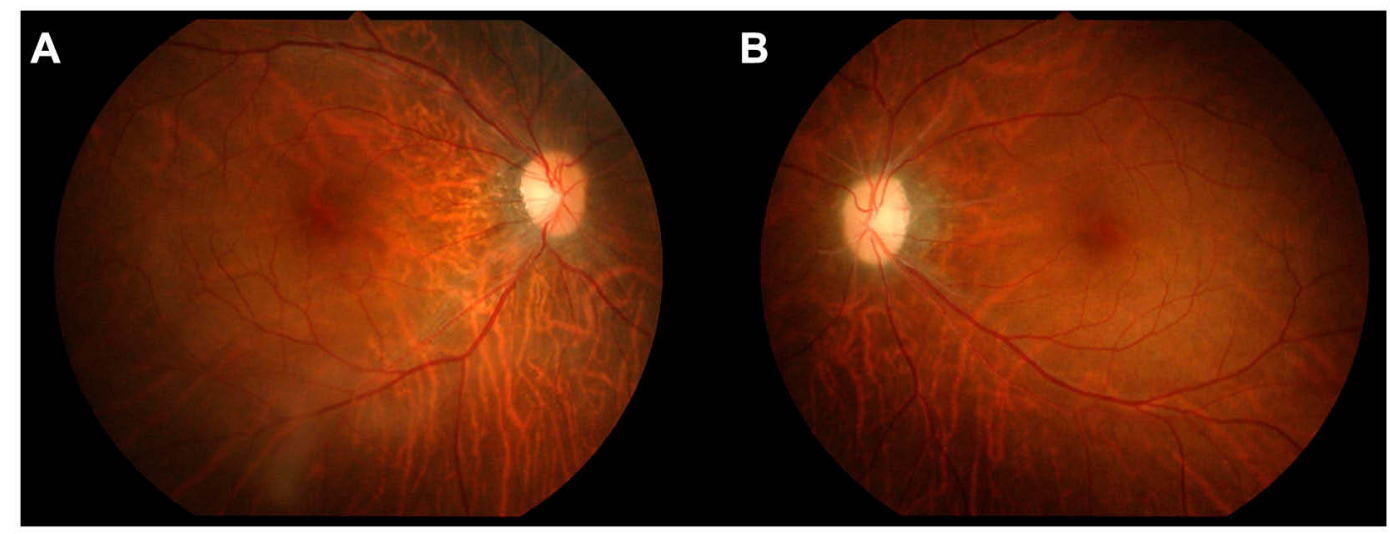

Figure 6 Color fundus photographs at the last presentation. Optic disc swelling had improved in (A) the right eye and (B) the left eye.

the initial symptoms are associated with increased intracranial pressure, such as headache $(73 \%)$ and nausea/vomiting $(45 \%) .{ }^{4}$ Although cranial nerve symptoms, such as visual impairment $(12 \%)$ and diplopia/eyelid drooping $(5 \%)$, are also observed, no cases of these being the first symptoms alone can be confirmed.

Although the diagnosis of meningeal carcinoma is based on comprehensive assessment of neurological symptoms, the presence of primary cancer, and cerebrospinal fluid findings, ${ }^{6}$ the definitive diagnosis is to prove the presence of tumor cells with cerebrospinal fluid cytology. The positive rate is about $50 \%$ in the first test, ${ }^{2,7}$ but can be increased to $91 \%$ by repeatexamination. ${ }^{2}$ Although signet-ring cell carcinoma was detected by the initial cytology in this study, at least two repeated tests are recommended if meningeal carcinoma is suspected and the initial cytology is negative. Olson reported that abnormal findings other than tumor cells are often obtained from the first cerebrospinal fluid test, such as an increase in pressure, an increase in protein, and a decrease in sugar. ${ }^{7}$ In addition, imaging examination, especially contrast-enhanced MRI with gadolinium, is useful for the diagnosis of meningeal carcinoma; however, enhancement effects of the sulcus, cerebral cistern, and subarachnoid space emerge after the progression. Since about $30 \%$ of meningeal carcinoma has no findings on MRI images, it is difficult to make a definitive diagnosis only with MRI images and it is necessary to make a comprehensive judgment based on clinical symptoms.

Treatment of meningeal carcinomatosis comprises radiotherapy, conventional systemic and intrathecal drug therapy, molecular targeted drugs, palliative treatment, or their combinations, but none has been established. Since meningeal carcinomatosis is a late symptom of cancer, there are many cases in which it is difficult to treat owing to deterioration of 
the general condition. Intrathecal chemotherapy has poor penetration into ocular tissues compared to the central nervous system, and its effect is not stable. ${ }^{8} \mathrm{VP}$ shunting is often effective for patients suffering from elevated intracranial pressure, and the performance status improves immediately after surgery. ${ }^{9-11}$ Although there is a risk of dissemination of tumor cells from the cerebrospinal fluid into the peritoneal cavity, it occurs very rarely. ${ }^{9}$ In addition, if systemic drug therapy is available, the transition to the peritoneal cavity is preferable and can be well controlled. In this case, symptoms of elevated intracranial pressure improved after VP shunt surgery, and the patient's quality of life (QOL) improved dramatically. There were no complications due to the VP shunt.

The prognosis for this disease is very poor. Patients with meningeal carcinomatosis due to solid cancer have a short life prognosis and, if not treated, median survival is 6-8 weeks. ${ }^{12}$ Treatments suitable for tumors, such as radiation therapy and drug treatment, lead to a slightly longer survival; nevertheless, the median survival is $2-8$ months. ${ }^{12,13}$

In conclusion, meningeal carcinomatosis should be suspected if papillary swelling with vision loss progresses rapidly even without a history of tumor. It is important to repeat procedures such as cytology and cerebrospinal fluid examination to allow earlier diagnosis and treatment. In addition, if there are symptoms of increased intracranial pressure that greatly reduce QOL, VP shunting should be actively performed to maintain QOL.

\section{Ethics and Consent Statement}

This case report was approved by the institutional review board of National Defense Medical College, and written informed consent to have the case details and any accompanying images published has been provided by the patient's next of kin.

\section{Disclosure}

The authors report no conflicts of interest in this work.

\section{References}

1. Posner JB, Chernik NL. Intracranial metastases from systemic cancer. Adv Neurol. 1978;19:579-592.

2. Wasserstorm WR, Glass JP, Posner JB. Diagnosis and treatment of leptomeningeal metastases from solid tumors: experience with 90 patients. Cancer. 1982;49:759-772. doi:10.1002/1097-0142(198202 15) 49:4<759::AID-CNCR2820490427>3.0.CO;2-7

3. Pavlidis N. The diagnostic and therapeutic management of leptomeningeal carcinomatosis. Ann Oncol. 2004;15 Suppl 4:iv285-iv291. doi:10.1093/annonc/mdh941

4. Gonzalez-Vitale JC, Garcia-Bunuel R. Meningeal carcinomatosis. Cancer. 1976;37:2906-2911. doi:10.1002/1097-0142(197606)37:6<2 906::AID-CNCR2820370648>3.0.CO;2-D

5. Lee JL, Kang YK, Kim TW, et al. Leptomeningeal carcinomatosis in gastric cancer. J Neurooncol. 2004;66:167-174. doi:10.1023/B:NEON. 0000013462.43156.f4

6. Amer MH, Al Sarraf M, Baker LH, Vaitkevicius VK. Malignant melanoma and central nervous system metastases. Cancer. 1978;42:660-668. doi:10.1002/1097-0142(197808)42:2<660::AIDCNCR2820420237>3.0.CO;2-E

7. Olson ME. Leptomeningeal metastasis from systemic cancer. A report of 47 cases. Trans Am Neurol Assoc. 1971;96:291-293.

8. Ellis W, Little HL. Leukemic infiltration of the optic nerve head. Am J Ophthalmol. 1973;75:867-871. doi:10.1016/0002-9394(73)90893-3

9. Omuro AM, Lallana EC, Bilsky MH, DeAngelis LM. Ventriculoperitoneal shunt in patients with leptomeningeal metastasis. Neurology. 2005;64:1625-1627. doi:10.1212/01.WNL.0000160396.69 050.DC

10. Lin N, Dunn IF, Glantz M, et al. Benefit of ventriculoperitoneal cerebrospinal fluid shunting and intrathecal chemotherapy in neoplastic meningitis: a retrospective, case-controlled study. J Neurosurg. 2011;115:730-736.

11. Nigim F, Critchlow JF, Kasper EM. Role of ventriculoperitoneal shunting in patients with neoplasms of the central nervous system: an analysis of 59 cases. Mol Clin Oncol. 2015;3:1381-1386. doi:10. 3892/mco.2015.627

12. Le Rhun E, Taillibert S, Chamberlain MC. Carcinomatous meningitis: leptomeningeal metastases in solid tumors. Surg Neurol Int. 2013;4:S265-S288. doi:10.4103/2152-7806.111304

13. Clarke JI, Perez HR, Jacks LM, Panageas KS, DeAngelis LM. Lewpto meningeal metastases in the MRI era. Neurology. 2010;74: 1449-1454. doi:10.1212/WNL.0b013e3181dc1a69

\section{Publish your work in this journal}

The International Medical Case Reports Journal is an international, peer-reviewed open-access journal publishing original case reports from all medical specialties. Previously unpublished medical posters are also accepted relating to any area of clinical or preclinical science. Submissions should not normally exceed 2,000 words or 4 published pages including figures, diagrams and references. The manuscript management system is completely online and includes a very quick and fair peer-review system, which is all easy to use. Visit http://www.dovepress.com/testimonials.php to read real quotes from published authors. 